\section{Projeto CÃOCER: uma Abordagem Educativa para a Prevenção de Cânceres em Animais}

\author{
The CÃOCER Project: an Educational Approach for Cancer \\ Prevention in Pet Animals
}

\section{RESUMO}

O projeto CÃOCER há quatro anos compreende atividades intra e extramurais à USP com a finalidade de conscientizar a população sobre a importância dos cânceres em animais, focando em sua prevenção. Foram realizados ciclos de palestras para alunos de graduação em Medicina Veterinária e profissionais. Participamos de feiras de ciência e exposições para divulgação à população de Pirassununga, e realizamos a I Cãopanha de Prevenção de Câncer em Animais em que foram feitos exames clínicos nos animais e distribuído material educativo referente à prevenção de câncer. Por fim, ainda objetivou-se realizar um levantamento estatístico da casuística de animais portadores de neoplasias atendidos no Hospital Veterinário da FMVZ no campus de Pirassununga da USP. Colaboraram com este projeto diversos alunos de graduação em Medicina Veterinária da FZEA-USP e alunos do ensino médio que obtiveram bolsas pelo Programa de Pré-Iniciação Científica da USP. Passados quatro anos desde seu início, o projeto atingiu seus objetivos iniciais e agora o intuito é expandi-lo. Para isso criamos um site (www.projetocaocer.com.br) que disponibiliza artigos sobre prevenção do câncer em animais. Pretendemos, também, aumentar as atividades extensionistas do projeto, junto à Associação Brasileira de Oncologia Veterinária e ao NAP de Oncologia Veterinária da Pró-Reitoria de Pesquisa da USP.

Palavras-chave: Prevenção do Câncer. Animais de Companhia. Atividades de Extensão.

\section{ABSTRACT}

The CÃOCER projetct started four years ago and comprised several intra and extramural activities with the purpose to aware people of the importance of cancer in animals, focusing mainly in cancer prevention. With this intent, we provided a series of lectures for undergraduates and professionals of veterinary medicine. In addition,

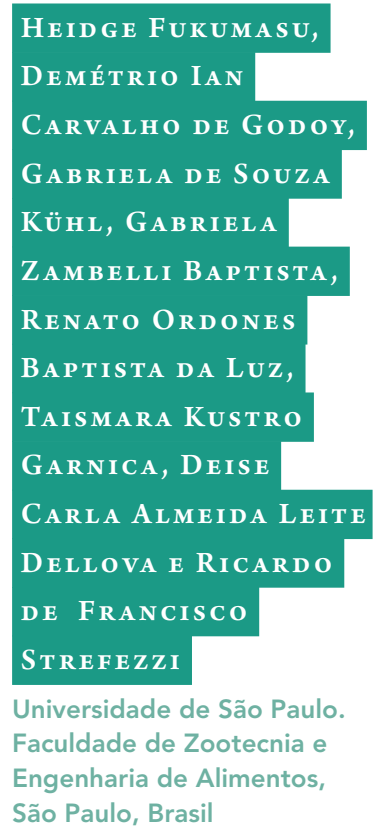


we participated in science fairs and exhibitions to disseminate the knowledge regarding cancer prevention for the population from Pirassununga, a city in the country of São Paulo State. Also, we conducted the first campaign of cancer prevention in animals named I Cãopanha of Cancer Prevention in Animals in which preventive physical clinical examinations were done on animals and educational material on cancer prevention was distributed. At last, it was also an objective of this work to perform a statistical survey of cancer cases in animals of Pirassununga. Some undergraduate students of Veterinary Medicine collaborated in this project with or without scholarships. Past four years since its inception, we believe that the project has achieved its initial goals and now our intention is to expand it. For this, we created a website (www.projetocaocer.com.br) that provides articles on cancer prevention in animals. We also wanted to increase the extension of the projetct activities, associating it with the Brazilian Association of Veterinary Oncology (ABROVET) and the Support Research Center in Veterinary Oncology from USP.

Keywords: Cancer Prevention. Pet Animals. Education.

\section{INTRODUÇÃO}

Câncer é uma das maiores preocupações na área médica atualmente e por isso é um dos principais assuntos estudados. Em 2005, no mundo morreram aproximadamente 7,6 milhões de pessoas e estima-se que em 2020 os números anuais sejam de 15 milhões [3]. Em 2007, de acordo com o National Center for Health Statistics, os cânceres foram a principal causa de morte nos EUA quando se trata de mulheres e homens dentro da faixa etária de 40 a 79 anos [5]. Em relação aos cães, o câncer é responsável por cerca de $50 \%$ das mortes dos animais que têm mais de 10 anos de idade [1]. Tomando como exemplo os tumores de mama, sabemos que estes são os mais frequentes em mulheres ( $32 \%$ ) assim como nas cadelas, onde correspondem a $52 \%$ de todos os diferentes tipos de câncer [4]. São diagnosticados com maior frequência em cadelas de meia idade ou mais velhas, sendo que 50\% são malignos [2]. Devido a mudanças no trato dos animais de companhia, associadas à melhora na dieta e programas de vacinação, os animais têm suas expectativas de vida aumentadas e, desta forma, estão mais predispostos a desenvolverem o câncer, assim como os humanos.

Também como em humanos, o tratamento e cura do câncer em cães e gatos são metas bastante difíceis, dependendo do quadro clínico do animal, em especial se há metástases. Portanto, é salutar estudar, aprimorar e divulgar medidas que previnam os quadros mais malignos de neoplasias. Dentre as opções mais comuns estão: dieta balanceada, exercícios, cuidado à exposição solar (neoplasias de pele), castração (neoplasias hormônio-dependentes como de mama e próstata e glândula ad-anal) e o diagnóstico preciso por parte do médico veterinário.

Portanto, a prevenção do câncer em humanos e animais de companhia é fundamental para a diminuição do número de mortes por essa doença, isso porque a detecção precoce aumenta muito as chances de cura. É muito importante que esse conceito seja divulgado e, assim, a população (tanto estudantes quanto leigos) seja 
conscientizada em relação às formas de detecção da doença e quais os procedimentos adequados para se evitar que seus animais venham a desenvolver o câncer.

Com esse intuito, o projeto CÃOCER (Figura 1) foi criado em 2010, tendo como principal objetivo promover a educação e conscientização sobre a importância do câncer em animais domésticos, sua prevenção e diagnóstico precoce para a população e estudantes de medicina veterinária de Pirassununga e região. Além disto, foi objeto deste projeto realizar levantamento da casuística regional das neoplasias em cães e gatos utilizando os casos atendidos no Hospital Veterinário da FMVZ-USP do campus de Pirassununga.

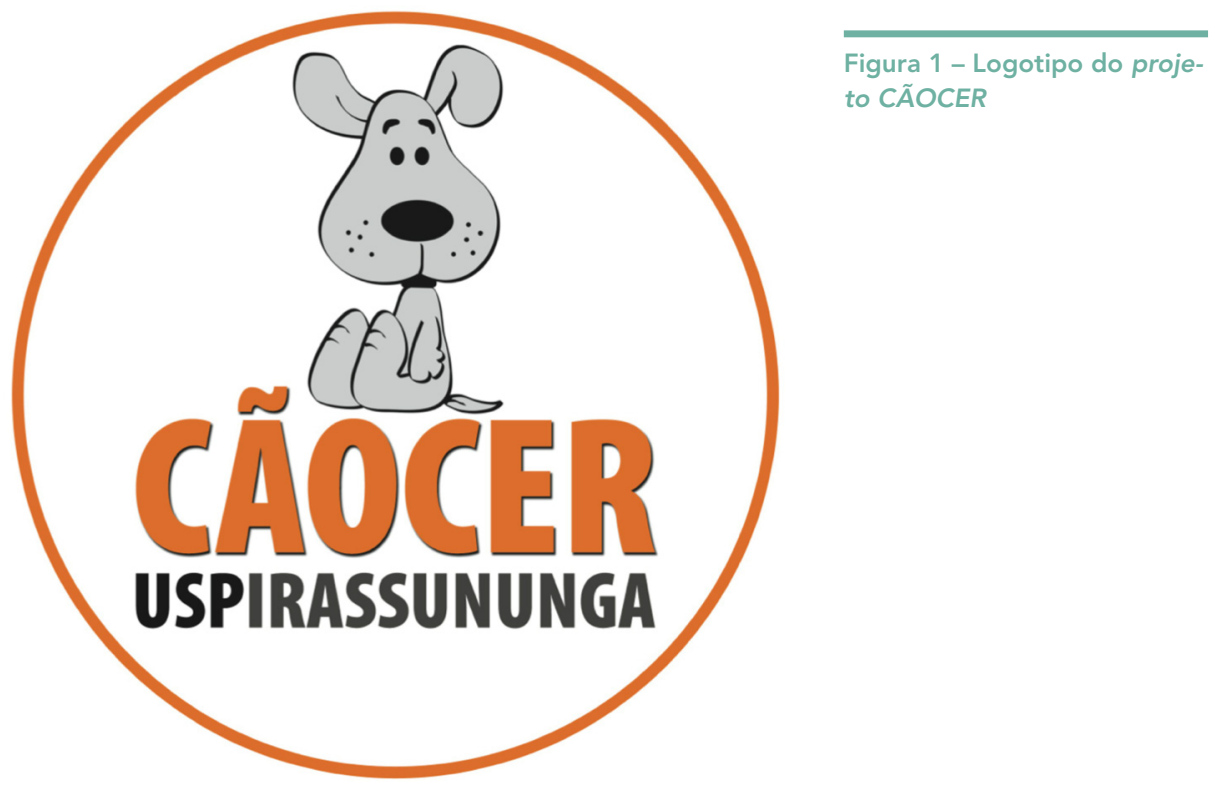

\section{MATERIAIS E MÉTODOS}

Para o desenvolvimento do projeto contou-se com a colaboração dos alunos do curso de Medicina Veterinária da FZEA-USP, campus de Pirassununga, participantes do projeto, bolsistas pelo Programa de Cultura e Extensão ou não. Foram confeccionados materiais de divulgação sobre câncer em animais e meios de prevenção. Além disso, foram realizados ciclos de palestras de atualização sobre o tema do projeto, contando com diversos professores, pesquisadores e profissionais da área. Também foi construído um site de internet para divulgação do projeto e seus ideais. Houve ainda a participação do grupo de estudantes e alunos associados ao projeto para realização de eventos extensionistas sobre o tema e como parte de outros eventos na cidade. Por fim, houve ainda o levantamento e análise da casuística de câncer em cães e gatos com os dados de prontuários do Hospital Veterinário da FMVZ-USP, situado no campus da USP de Pirassununga. Para a realização do estudo retrospectivo de neoplasias em cães e gatos atendidos no Hospital Veterinário da USP de Pirassununga, 
foram recolhidos dados a partir dos prontuários dos animais atendidos no período de 2007 até 2011. Durante a análise dos prontuários, foram registrados dados como raça, sexo e tipo de neoplasia. Os dados foram então organizados em tabelas e gráficos para realização de uma análise comparativa. Posteriormente tais dados também foram utilizados com objetivo científico, com a finalidade de caracterizar a casuística de neoplasias do Hospital Veterinário da Faculdade de Medicina Veterinária e Zootecnia da USP campus de Pirassununga.

\section{RESULTADOS E DISCUSSÃO}

O Projeto Cãocer foi iniciado em meados de 2010 com o primeiro ciclo de palestras sobre temas relevantes à prevenção de câncer em animais, como pode ser visto na Tabela 1. Nos anos seguintes, outras palestras foram realizadas periodicamente no anfiteatro central do campus de Pirassununga da USP contando com a participação dos alunos do curso de Medicina Veterinária tanto da USP como de outras faculdades e universidades da região como Uniararas - Fundação Hermínio Ometto, Faculdade Anhanguera, Unifeob, Unicastelo, entre outras. Além dos alunos, foi realizado convite aos médicos veterinários de Pirassununga e região, com boa participação dos profissionais, que permitiu a discussão de um convênio com a Associação de Médicos Veterinários de Leme, cidade vizinha a Pirassununga.

Tabela 1 - Palestras técnicas ocorridas nos ciclos de palestras CÃOCER

\begin{tabular}{|c|c|c|}
\hline TÍTULO DA PALESTRA & PALESTRANTE & DATA \\
\hline $\begin{array}{c}\text { Conceitos Básicos em } \\
\text { Neoplasia }\end{array}$ & Prof. Dr. Ricardo F. Strefezzi & $20 / 10 / 2010$ \\
\hline $\begin{array}{c}\text { Bases Moleculares do } \\
\text { Câncer }\end{array}$ & Prof. Dr. Heidge Fukumasu & $10 / 11 / 2010$ \\
\hline Epidemiologia do Câncer & $\begin{array}{c}\text { Profa. Dra. Trícia M. S. F. } \\
\text { Oliveira }\end{array}$ & $25 / 11 / 2010$ \\
\hline $\begin{array}{l}\text { Prevenção e Diagnóstico } \\
\text { do Câncer em Animais }\end{array}$ & Profa. Dra. Renata A. Sobral & $25 / 05 / 2011$ \\
\hline $\begin{array}{c}\text { A Experiência em Ser } \\
\text { Oncologista Veterinária: } \\
\text { Casos de Sucesso }\end{array}$ & Profa. Dra. Renata A. Sobral & $25 / 05 / 2011$ \\
\hline
\end{tabular}


O Uso da Imuno-

historiquímica para o Diagnóstico, Prognóstico e

Tratamento das Neoplasias
Profa. Dra. Maria L.Z. Dagli

$18 / 08 / 2011$

Quimioterapia

Antineoplásica: Mais uma Arma Contra o Câncer em

Cães e Gatos
Prof. Dr. Andrigo B. De Nardi

$18 / 10 / 2011$

$17 / 05 / 2012$

Profa. MS. Adriana Tomoko

Nishiya

$13 / 11 / 2012$

Lucas Campos de Sá

Rodrigues cológico

Princípios em Cirurgia

Oncológica

$(13 / 11 / 2012$

Abordagens Inovadoras

Contra o Câncer:

Quimioprevenção e Novas

Terapias

26/11/2012

Uma outra atividade importante do projeto foi o aprimoramento técnico dos alunos participantes, necessário, por exemplo, para a criação do material para divulgação sobre o câncer em animais, assim como meios para seu diagnóstico e prevenção, material este todo criado pelos alunos do projeto e revisados pelos docentes (Figura 2). Foram criados folders com os temas: O que fazer para prevenir câncer no seu animal?, Câncer em animais domésticos: diagnóstico e tratamento. e O que é câncer?. Todo o material preparado foi utilizado para confecção de folders, banners e colocado ainda no site do projeto (Figura 3), disponibilizado gratuitamente a quem tiver interesse.

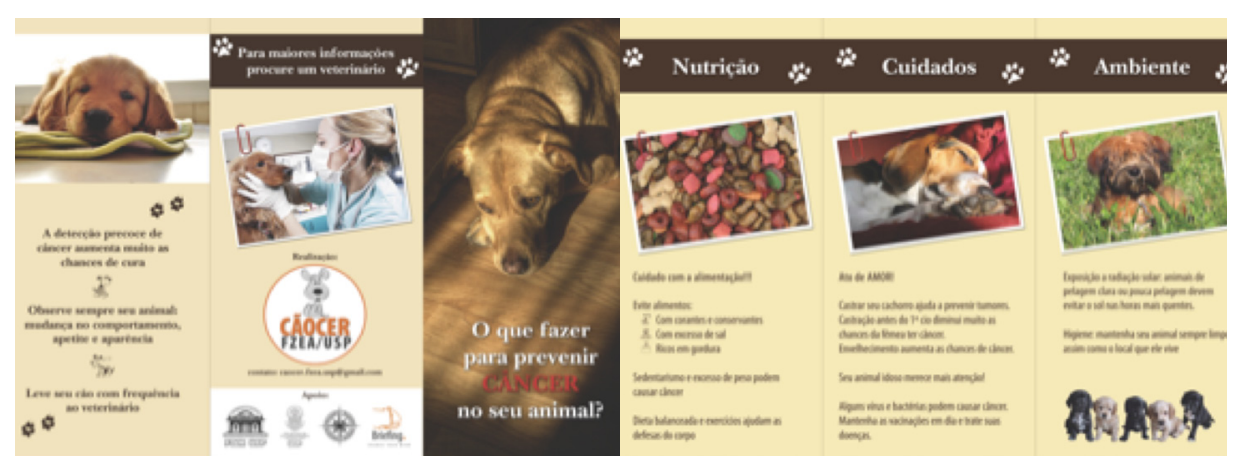

Figura 2 - Folders educativos preparados pelos alunos participantes do projeto e revisados pelos docentes. 


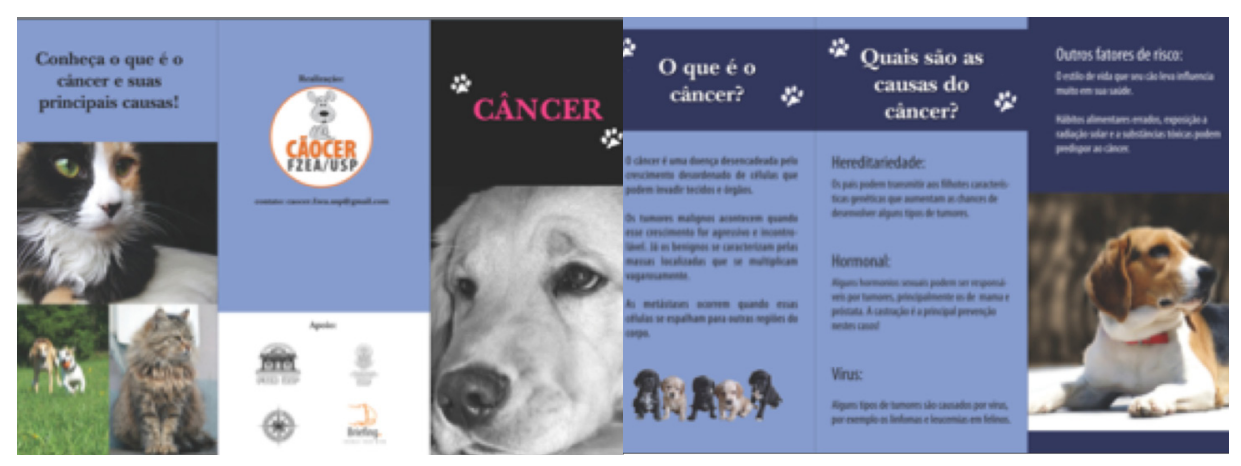

Figura 3 - Site do Projeto CÃOCER, disponível em: www.projetocaocer.com.br

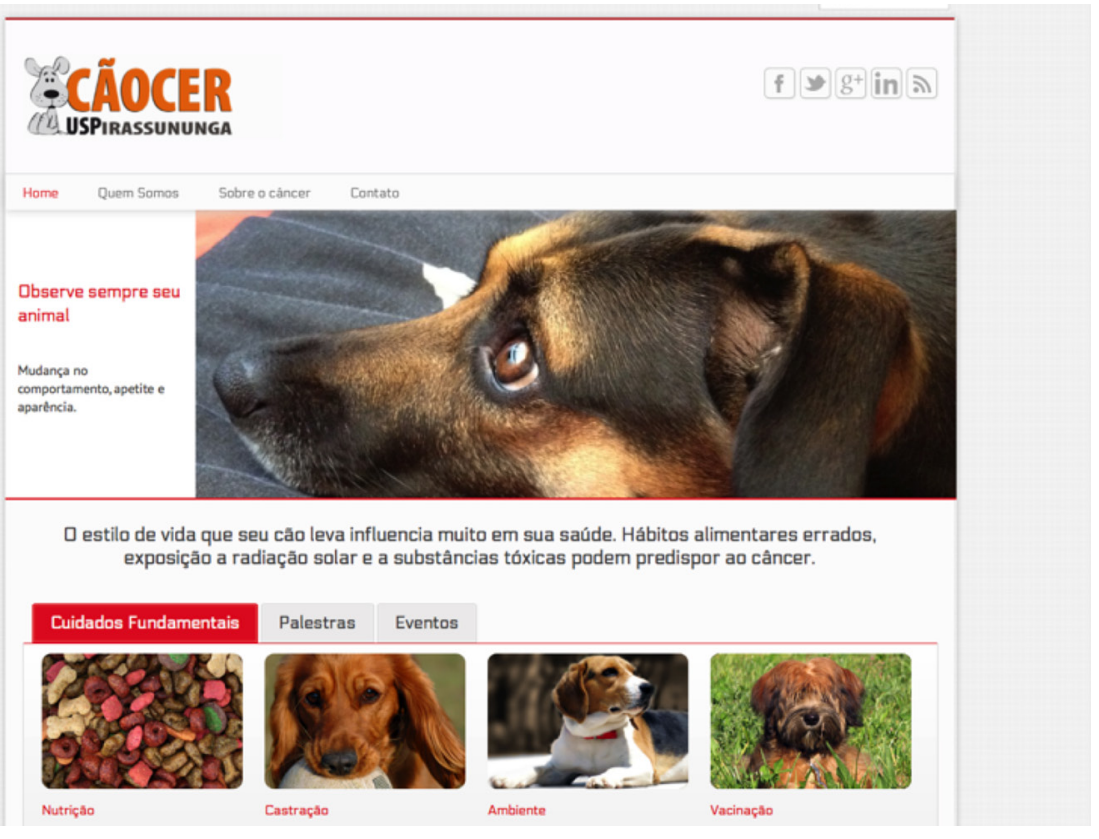

Outra forma de contribuir para a divulgação de informações sobre a importância do câncer em animais e principalmente da sua prevenção foi a participação do grupo Cãocer em eventos na cidade de Pirassununga como feiras de eventos e feiras de ciências de colégios da região, como a Exposhow 2011, Feira de Ciências do Colégio Objetivo, Dia de Responsabilidade Social promovido pela FATECE-Pirassununga, dentre outras. $\mathrm{O}$ intuito deste tipo de atividade foi divulgar à população em geral os princípios do projeto e sua importância, tendo como foco desde crianças a adultos. Além deste tipo de participação educativa, o grupo de alunos do projeto Cãocer participou das duas Campanhas de Prevenção de Câncer de Mama em Cadelas (2012 e 2013) no parque do Ibirapuera em São Paulo, organizado pelo Hospital Onco Cane, sob responsabilidade da M.V. Renata Afonso Sobral, colaboradora do nosso projeto. 


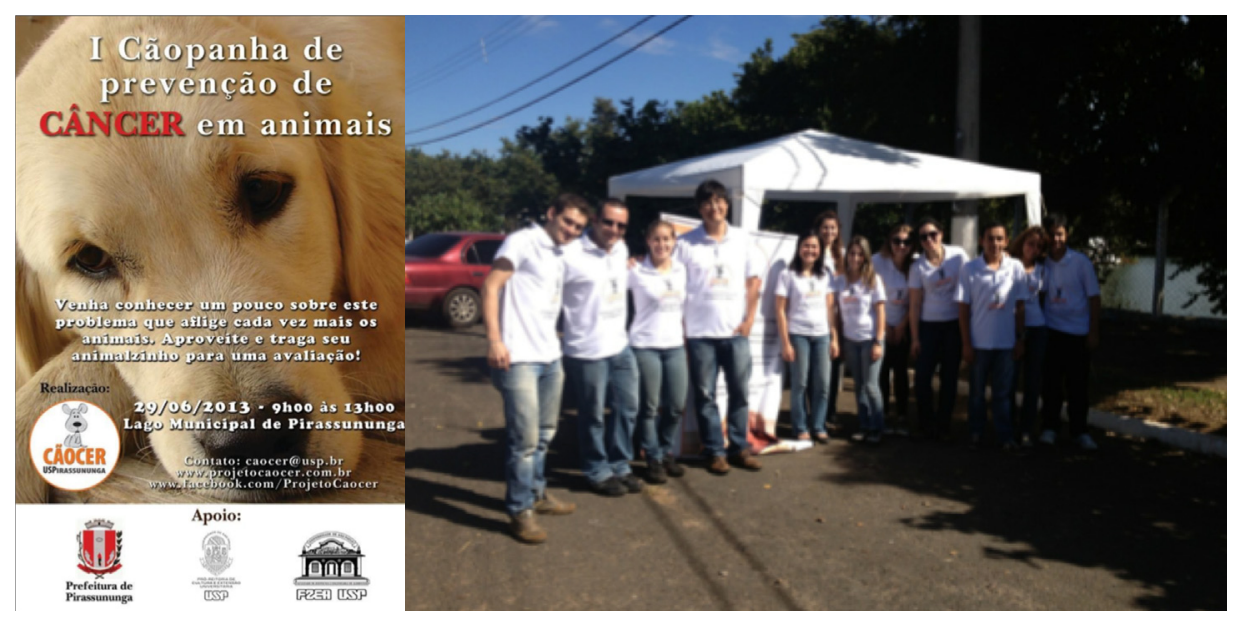

Figuras 4 e 5 - I Cãopanha de Prevenção de Câncer em Animais
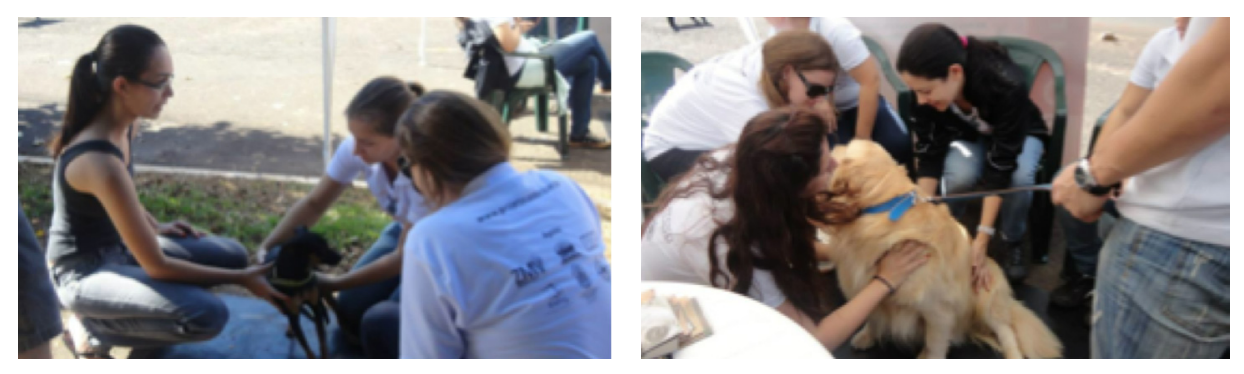

Em 29 de junho de 2013 foi realizada a I Cãopanha de Prevenção de Câncer em Animais de Pirassununga, com a participação de docentes e alunos do curso de Medicina Veterinária da FZEA-USP (Figuras 4 e 5 ). Neste evento, em pouco mais de quatro horas, foram abordadas mais de 50 pessoas, com realização de exame físico preventivo em 33 animais, dos quais sete apresentaram alguma formação tumoral aparente, em pele ou glândula mamária, sendo encaminhados às clínicas veterinárias da cidade. Este atendimento foi realizado pelos docentes e médicos veterinários associados ao projeto, sempre com os alunos de graduação em Medicina Veterinária acompanhando as atividades. Essas formações tiveram suas características anotadas quanto o tamanho, localização, consistência, tipo de superfície, tipo de base, se eram subcutâneas ou cutâneas e se apresentavam ou não aderência, alopecia, eritema, ulceração, sensibilidade e hiperpigmentação, a temperatura do animal também foi verificada.

Como mencionado acima, outro objetivo do projeto foi o de levantar a casuística de animais portadores de neoplasias na região de Pirassununga. Para isso, foi realizado acordo científico com os responsáveis pelo Hospital Veterinário da FMVZ-USP situado no campus de Pirassununga. A partir do levantamento de cinco anos, entre 2007 e 2011, foram avaliados 2796 prontuários de animais atendidos no período com 286 diagnósticos de suspeita de neoplasias. Maiores informações sobre este levantamento serão publicadas oportunamente. 


\section{CONSIDERAÇÕES FINAIS}

Somando-se todas as atividades descritas acima realizadas no âmbito do projeto Cão$c e r$, acreditamos que o objetivo educativo e extensionista do projeto foi atingido. Pretendemos manter o projeto ativo e ampliar suas ações com a realização da II Cãopanha de Prevenção e mais palestras técnicas e educativas. Esperamos que, futuramente, tenhamos redução no número de óbitos e incremento no diagnóstico e tratamento precoce de câncer em animais na região, o que certamente contribuirá para maior sobrevida e qualidade de vida dos animais de companhia de nossa região

\section{REFERÊNCIAS}

[1] BAEK, S. J.; MCENTEE, M. F.; LEGENDRE, A. M. Review paper: Cancer chemopreventive compounds and canine cancer. Veterinary pathology, v. 46, n. 4, p. 576-88, jul. 2009. DOI: 10.1354/vp.08-VP-0238-B-REV

[2] HELLMÉN, E. Complex mammary tumours in the female dog: a review. The Journal of dairy research, v. 72 Spec No, p. 90-7, jan. 2005. DOI:10.1017/ Soo2202990500124X

[3] INCA. Estimativa 2012 : incidência de câncer no Brasil. Instituto Nacional de Câncer José Alencar Gomes da Silva, 2011.

[4] QUEIROGA, F.; LOPES, C. Tumores mamários caninos, pesquisa de novos factores de prognóstico. RPCV, n. 543, p. 119-127, 2002.

[5] SIEGEL, R.; NAISHADHAM, D.; JEMAL, A. Cancer statistics, 2013. CA: a cancer journal for clinicians, v. 63, n. 1, p. 11-30, jan. 2013. DOI: 10.3322/ caac. 21166

\section{AGRADECIMENTOS}

Gostaríamos de agradecer à Empresa Briefing Comunicação, na pessoa da Sra. Alice Ordones, pela colaboração no design e preparo do material educativo e do site. Também agradecemos à Faculdade de Zootecnia e Engenharia de Alimentos pelo apoio incondicional ao projeto. Agradecemos à FMVZ-USP pela colaboração no levantamento da casuística dos casos de câncer. Por fim, gostaríamos de agradecer a todos aqueles que colaboraram para a realização do projeto. 
HEIDGE FUKUMASU professor associado do Departamento de Medicina Veterinária da Faculdade de Zootecnia e Engenharia de Alimentos da Universidade de São Paulo (FZEA-USP), coordenador do Laboratório de Oncologia Comparada e Translacional da USP e idealizador do Projeto Cãocerde Extensão e Pesquisa-e-mail:fukumasu@usp.br

Deise CARLA ALMEIDA LEITE DELlova professora doutora do Departamento de Medicina Veterinária da Faculdade de Zootecnia e Engenharia de Alimentos da Universidade de São Paulo (FZEA-USP)

RICARDO DE FRANCISCO STREFEZZI professor associado do Departamento de Medicina Veterinária da Faculdade de Zootecnia e Engenharia de Alimentos da Universidade de São Paulo (FZEA-USP)

DEMÉTRIO IAN CARVALHO DE GODOY graduando em Medicina Veterinária da Faculdade de Zootecnia e Engenharia de Alimentos da Universidade de São Paulo (FZEA-USP)

GABRIELA DE SOUZA KÜHL graduanda em Medicina Veterinária da Faculdade de Zootecnia e Engenharia de Alimentos da Universidade de São Paulo (FZEA-USP)

RENATO ORDONES BAPTISTA DA LUZ graduando em Medicina Veterinária da Faculdade de Zootecnia e Engenharia de Alimentos da Universidade de São Paulo (FZEA-USP)

TAISMARA KUSTRO GARNICA graduanda em Medicina Veterinária da Faculdade de Zootecnia e Engenharia de Alimentos da Universidade de São Paulo (FZEA-USP)

GABRIELA ZAMBELLI BAPTISTA graduanda em Engenharia de Biossistemas da Faculdade de Zootecnia e Engenharia de Alimentos da Universidade de São Paulo (FZEA-USP) 